\title{
镜像疗法联合常规言语训练治疗急性脑梗死后 皮质下失语疗效观察
}

\author{
陈庆梅, 蔡秀英, 柯 俊, 沈丹, 范可可, 杨卫新, 李 莉, 方 琪 “ \\ 苏州大学附属第一医院, 江苏苏州 215000 \\ *通信作者:方琪,E-mail :fangqi_008@126.com
}

收稿日期:2020-02-28; 接受日期:2020-04-08

基金项目:国家自然科学基金青年项目 (81701669); 江苏省社会发展一重点病种规范化诊疗项目 (BE2016670); 江苏省自然科学基金青年项目 (BK20170368)

DOI : 10.3724/SP.J.1329.2020.03010

开放科学 (资源服务)标识码 (OSID):

摘要 目的: 观察镜像疗法联合常规言语训练治疗急性脑梗死后皮质下失语的临床疗效。方法: 将纳入急性 脑梗死后皮质下失语患者 34 例随机分成治疗组和对照组各 17 例。2 组患者均于发病后第 7 天开始接受 2 周的常规言语训䋆, 治疗组在常规言语治疗基础上加用镜像治疗, 对照组仅给予常规言语治疗, 评估 2 组 治疗前、病程第 3 周及病程第 12 周失语商 (AQ)、美国国立卫生研究院卒中量表 (NIHSS) 和改良 Rankin 量 表 $(\mathrm{mRS})$ 。以 3 个月 $\mathrm{AQ}$ 提高 $\geqslant 30$ 分为言语功能明显改善, 改良 $\mathrm{mRS} 0 \sim 2$ 分为神经功能预后良好。结果: 治疗前 2 组各项指标比较差异无统计学意义 $(P>0.05)$; 经过 2 周治疗后, 2 组病程第 3 周和第 12 周 NIHSS、 $\mathrm{AQ} 、 \mathrm{AQ}$ 各项(自发言语、听理解、复述及命名)及 $\mathrm{mRS}$ 得分均较治疗前改善, 差异有统计学意义 $(P<0.05)$; 病程第 3 周和第 12 周治疗组的 AQ、自发言语、听理解、复述、命名、NIHSS 及 $\mathrm{mRS}$ 改善优于对照组 $(P<$ $0.05)$; 治疗组的言语功能明显改善率 (82.35\%) 较对照组 (35.29\%) 更为显著 $(P=0.013)$ 。校正混杂因素后, 千 预方式及年龄与言语功能明显改善相关 $(P<0.05)$ 。结论: 镜像疗法结合常规言语训练有助于急性脑梗死后 皮质下失语患者言语和神经功能的康复, 同时促进患者 3 个月言语功能的明显改善。

关键词急性脑梗死; 皮质下失语; 镜像疗法; 常规言语训练; 言语功能

镜像疗法又称镜像视觉反馈疗法,已经被证实 在提高脑卒中偏痽患者的上肢运动功能及减轻复 杂性区域疼痛综合征患者的疼痛方面疗效显著 ${ }^{[1-2]}$, 而针对失语症的治疗研究较少。近期有研究表明, 基于镜像神经元理论的手动作观察可以改善慢性 期失语症患者言语功能 ${ }^{[3-7]}$ 。然而, 这些研究主要针 对的是慢性期皮层性失语症的患者,而关于镜像疗 法用于治疗急性脑梗死后皮质下失语的研究鲜有 报道。因此, 本研究旨在探索镜像疗法联合常规言 语训练对急性脑梗死后皮质下失语患者言语功能 及神经功能恢复的影响。

\section{1 临床资料}

\section{1 病例选择标准}

1.1.1 纳人标准 (1) 人选患者均符合 2018 年中国 急性缺血性脑卒中诊治指南制定的脑梗死诊断标 准; (2) 病程 $<14 \mathrm{~d}$; (3) 符合皮质下失语临床表现; (4) 经西方失语症成套测验 (Western Aphasia Battery, $\mathrm{WAB}$ ) 且失语商 (aphasia quotient, $A Q$ ) 得分 $<$ 93.8 分 $^{[8]}$; (5) 弥散加权成像为明确的左半球言功能 区皮质下结构单一病灶 (卒中部位主要包括基底 核一内囊区、丘脑及脑室周围白质等部位); (6) 教育 年限 $\geqslant 5$ 年; (7) 经利手标准化判定为右利手; (8) 发病

引用格式:陈庆梅,暴秀英,柯俊,等. 镜像疗法联合常规言语训练治疗急性脑梗死后皮质下失语疗效观察 $[\mathrm{J}]$. 康复学报, 2020,30(3): 220-225.

CHEN Q M,CAI X Y,KE J, et al. Therapeutic effect of mirror therapy combined with conventional speech training on subcortical aphasia after acute cerebral infarction [J]. Rehabilitation Medicine, 2020,30(3):220-225.

DOI : $10.3724 /$ SP.J.1329.2020.03010 
前无言语异常; (9) 母语为汉语; (10) 年龄 18 岁以上; (11) 患者知情同意并签署知情同意书。

1.1.2 排除标准 (1) 脑卒中后病情严重或伴有意 识障碍不能配合检查; (2) 有进行静脉内溶栓或者 血管内治疗患者; (3) 伴严重感知或认知功能障碍 (non-language cognitive function assessment scale $>$ $70)^{[9]}$; (4) 伴精神障碍史或抑郁病史; (5) 妊娠、近期 心肌梗死病史、严重心功能不全、重要脏器严重功 能障碍、难以控制的高血压、合并癫痡者; (6) 视力、 听力障碍者; (7) 发病前有构音障碍、口吃等语言障 碍; (8) 病灶累及或双侧半球者; (9) 有 MRI 检查禁忌 证及不合作者。

\section{2 一般资料}

本研究采用随机对照研究方法, 对评定人员采取 盲法。前瞻性地纳入苏州大学附属第一医院 2018 年 1 月一 2020 年 3 月住院治疗的急性脑梗死后皮质下 失语症患者 34 例。所有实验设计符合人体试验委员 会制定的伦理学标准, 所有患者纳人前均签署知情 同意书(临床试验注册号:ChiCTR1900028518)。根据 CHEN 等[10]研究结果, 得出本研究最小样本量为 24 例。共纳人 40 例患者, 其中有 6 例患者失访, 完成 随访者 34 例, 治疗组和对照组各 17 例。2 组的性 别、年龄、教育年限、既往史 [高血压、糖尿病、冠状 动脉粥样硬化性心脏病 (简称冠心病)、脑卒中及房 颤]及卒中部位等一般资料经统计学分析, 差异均 无统计学意义 $(P>0.05)$, 见表 1 。

表 12 组一般资料比较

Table 1 Comparison of general data between two groups

\begin{tabular}{|c|c|c|c|c|c|c|c|c|c|c|}
\hline \multirow{2}{*}{ 组 别 } & \multirow{2}{*}{ 例数 } & \multicolumn{2}{|c|}{ 性别 $(n, \%)$} & \multirow{2}{*}{ 年龄 $(\bar{x} \pm s$, 岁 $)$} & \multirow{2}{*}{$\begin{array}{c}\text { 教育年限 } \\
(\bar{x} \pm s, \text { 年 })\end{array}$} & \multirow{2}{*}{$\begin{array}{c}\text { 高血压 } \\
(n, \%)\end{array}$} & \multirow{2}{*}{$\begin{array}{c}\text { 煻尿病 } \\
(n, \%) \\
\end{array}$} & \multirow{2}{*}{$\begin{array}{l}\text { 冠心病 } \\
(n, \%)\end{array}$} & \multirow{2}{*}{$\begin{array}{c}\text { 脑卒中 } \\
(n, \%)\end{array}$} & \multirow{2}{*}{ 房顫 $(n, \%)$} \\
\hline & & 男 & 女 & & & & & & & \\
\hline 对照组 & 17 & $12(70.59)$ & $5(29.41)$ & $62.18 \pm 11.64$ & $6.65 \pm 2.32$ & $10(58.82)$ & $4(23.53)$ & $2(11.76)$ & $6(35.29)$ & $3(17.64)$ \\
\hline 治疗组 & 17 & $16(94.12)$ & $1(5.88)$ & $55.88 \pm 11.76$ & $8.06 \pm 2.33$ & $11(64.71)$ & $3(17.64)$ & $2(11.76)$ & $1(5.88)$ & $0(0.00)$ \\
\hline$\chi^{2 / t}$ 值 & & & & -1.569 & 1.771 & & & & & \\
\hline$P$ 值 & & \multicolumn{2}{|c|}{0.175} & 0.127 & 0.086 & 1.000 & 1.000 & 1.000 & 0.085 & 0.227 \\
\hline
\end{tabular}

组 别 例数 基底核一内囊区 $(n, \%)$ 丘脑 $(n, \%)$ 脑室旁白质 $(n, \%)$ 基底核十丘脑 $(n, \%)$ 基底核十脑室旁白质 $(n, \%)$

\begin{tabular}{ccccccc}
\hline 对照组 & 17 & $6(35.29)$ & $1(5.88)$ & $3(17.64)$ & $2(11.76)$ & $6(35.29)$ \\
治疗组 & 17 & $6(35.29)$ & $1(5.88)$ & $4(23.53)$ & $1(5.88)$ & $7(41.18)$ \\
$\chi^{2} / t$ 值 & & & & & 1.000 & 1.000 \\
$P$ 值 & & 1.000 & 1.000 & 1.000 & \\
\hline
\end{tabular}

\section{2 研究方法}

\section{1 分组}

人组患者按照电脑生成的数字表随机分为治 疗组和对照组,采用完全随机化原则 (1:1), 电脑随 机生成单双号分成 2 组: 单号人组治疗组, 双号人 对照组。对照组给予常规言语治疗,治疗组在常规 言语训练的基础上加用镜像治疗。(1) 常规言语训 练: 所有患者在发病后第 7 天, 根据患者语言功能 评定的结果制定相应的听、说、读、写治疗方案, 由 治疗师对患者进行一对一的语言训练。如综合应用 Schuell 失语症刺激疗法、交流效果促进法、去除阻 断法等教会患者言语表达技能,行言语、命名、复述、 朗读、书写等训练。常规言语功能训练每次 $30 \mathrm{~min}$, 每日 2 次, 每周 $5 \mathrm{~d}$, 疗程 2 周。(2) 镜像疗法: 治疗 组于发病后第 7 天开始辅以镜像治疗, 每次训练 30 $\min$, 每周 $5 \mathrm{~d}$, 疗程 2 周。本研究镜像治疗采用苏州 明思特医疗科技有限公司生产的镜像神经元康复 训练系统 V1.0(MNST-V1.0)进行。选择系统中语言 组治疗部分, 该部分有 320 个与日常生活手部活动 相关的动作视频资料。每个动作视频均先后以 3 种 形式播放呈现给患者, 首先播放说出动作的口型配 合该动作的语音, 然后为执行动作的手势动作配合 语音,最后是口型结合手势动作同时配合语音。动 作的每种形式播放时长为 $3 \mathrm{~s}$, 每个动作视频分 3 种 形成共计播放 $9 \mathrm{~s}$ 。如打电话、用杯子喝水、读报纸、 切西瓜、吹蜡烛、用钥匙开门、用笔写字、剥花生等, 根据患者所处的康复阶段, 给予不同难度的手动作 视频。

\section{2 随访}

按卒中急性期处理指南和二级预防指南标准, 对所有患者进行急性期治疗和二级预防治疗。实验 
结束后根据每位患者实际功能评估情况安排继续 相应的常规康复治疗。人组患者按病程第 3 周及病 程第 12 周进行面对面随访, 详细了解患者的康复情况。

\section{3 统计学方法}

采用 SPSS 22.0 软件进行数据分析。所有数据 经过正态性检验,符合正态分布者以 $(\bar{x} \pm s)$ 表示, 2 组组间比较符合正态分布的计量资料采用 $t$ 检验, 不符合正态分布的计量资料Mann-Whitney $U$ 检 验,符合正态分布计数资料采用 $\chi^{2}$ 检验, 组内各时 间点比较采用Friedman 秩和检验方法。为校正混杂 因素影响, 对 3 个月的言语明显改善进行二元 $L o-$ gistic 回归分析。 $P<0.05$ 为差异具有统计学意义。

\section{3 结 果}

\section{1 疗效判定标准}

采集所有患者治疗前、病程第 3 周及病程第 12 周的疗效评估。(1) 自发言语:包括信息内容和流畅 度; (2) 听理解: 包括是/否问题、听辨认和指令执行; (3) 复述; (4) 命名: 包括物品命名、列名、完成句子和 反应命名。

$A Q^{[11-12]}=($ 自发言语十口语理解 $/ 20+$ 复述 $/$ $10+$ 命名, 10$) \times 2$

采用美国国立卫生研究院脑卒中量表 (National Institutes of Health Stroke Scale,NIHSS) 和改良Rankin 量表 (Modified Rankin Scale, mRS) 评估神经功能。 以 3 个月 $\mathrm{AQ}$ 提高 $\geqslant 30$ 分为言语功能明显改善 ${ }^{[13]}$ 。 神经功能预后的判定: 以 3 个月 $\mathrm{mRS} 0 \sim 2$ 分为神 经功能预后良好, $m R S>2$ 分为神经功能预后不良。 评定由同一名对分组不知情的言语治疗师和另一 名临床医师分别完成。

\subsection{2 组治疗前后各项指标比较}

(1) 2 组病程第 3 周及病程第 12 周 $\mathrm{AQ} 、 \mathrm{AQ}$ 各 项 (自发言语、听理解、复述及命名)、NIHSS 及 mRS 得分均较治疗前改善, 差异具有统计学意义 $(P<$ 0.05 ), 见表 2; (2) 治疗组病程第 3 周及病程第 12 周 AQ、自发言语、听理解、复述、命名、NIHSS 及 $\mathrm{mRS}$ 改 善优于对照组 $(P<0.05)$, 见表 2 ; (3) 治疗组的言语 功能明显改善率 $(82.35 \%)$, 较对照组 (35.29\%) 更 为显著 $(P=0.013)$, 见表 2 。

\section{3 多因素 Logistic 回归分析}

(1) 将性别、年龄、教育年限、高血压等基线资料 进行共线性分析, 发现 VIF 值均 $<10$, 各自变量之 间不存在共线性,再将单一基线资料纳人单因素 Logistic 回归方程中, 发现性别、年龄及干预方式与
言语功能明显改善相关 $(P<0.05)$ 。(2) 再将性别、年 龄及干预方式纳人多因素Logistic 回归方程, 发现 只有干预方式和年龄与言语功能明显改善存在相 关性 $(P<0.05)$, 见表 3。

\section{4 讨 论}

皮质下失语症是由各种原因 (如出血、梗死、脓 肿、变性等)引起的纹状体一内囊结构、丘脑和/或脑 室周围白质 (不包括紧邻各脑区的皮质下白质) 的 局限性病变 ${ }^{[14-21]}$ 。主要表现为词汇记忆障碍 ${ }^{[22]}$, 复述 能力相对保留 ${ }^{[17,23]}$ 。既往研究认为, 皮质下结构在语 言运动控制和语言形成中的作用突出 ${ }^{[24-25]}$ 。而皮质 下失语在脑卒中后发生率较高, 有研究表明 $40 \%$ $70 \%$ 皮质下脑血管病患者出现了皮质下失语综合 征 ${ }^{[26-27]}$ 。皮质下失语不但使患者进一步致残, 且影响 患者的情绪及运动障碍的恢复。因此分析其言语障 碍的特征并予以针对性康复治疗, 对患者的全面康 复有着重要的意义。

目前, 有关皮质下失语症的发病机制尚无明确 定论, 主流的假说包括: (1) 神经机能联系失能 (diaschisis), 也称受损伤远隔部位的生理功能障碍; (2) 皮质下结构直接参与语言加工过程, 皮质下结构损 害可直接导致失语; (3) 皮质下病变导致皮层语言区 的低代谢及低灌注, 如血肿、压迫、缺血半暗带等的 影响; (4) 皮层语言区断离; (5) 皮质下病变所致的皮 层调节语言功能的释放 ${ }^{[14]}$ 。皮质下失语是以上一种 或多种机制综合作用的结果 ${ }^{[28]}$ 。而针对皮质下失语 的药物或言语康复治疗鲜见报导。且在我国绝大部 分急性脑梗死的患者就诊于神经内科, 早期的康复 手段很难介人。因此, 早期皮质下失语的康复潜力 非常有限, 而镜像疗法具有便利、个性化且无创伤 及安全的特点, 该项康复治疗措施更容易在国内早 期言语康复中推广运用。

基于镜像神经元理论的镜像疗法, 在中枢神经 系统内部可以直接激活人脑镜像神经元系统 (mirror neuron system, MNS), 促进了语言功能区重组, 因此可以表现为语言的运动表达能力、语义理解及 运动学习能力的提高, 最终改善了语言功能, 是 一种自上而下的言语康复的方法。同时, MNS 参与 动作的理解、模仿、共情、意图共鸣、心理理论及社 会认知等认知活动 ${ }^{[29]}$ 。因此, 近年来有研究将镜像 神经元理论应用于失语症的治疗取得了显著的疗 效 ${ }^{[6-7,10]}$ 。

本研究结果显示, 与常规言语治疗比较镜像疗 
表 22 组治疗前后各项指标比较

Table 2 Comparison of indicators before and after treatment between two groups

\begin{tabular}{|c|c|c|c|c|c|c|c|c|c|}
\hline 组 别 & 例数 & 时间 & $\mathrm{AQ}$ & \multicolumn{2}{|l|}{ 自发言语 } & \multicolumn{2}{|l|}{ 听理解 } & \multicolumn{2}{|c|}{ 复述 } \\
\hline \multirow{3}{*}{ 对照组 } & \multirow{3}{*}{17} & 治疗前 & $39.500(1.900,67.700)$ & \multicolumn{2}{|l|}{$7.588(0.000,14.000)$} & \multicolumn{2}{|c|}{$3.894(0.2000,7.000)$} & \multicolumn{2}{|c|}{$4.179(0.750,6.375)$} \\
\hline & & 病程第 3 周 & $49.194(4.700,83.300)^{1)}$ & \multicolumn{2}{|c|}{$9.529(2.000,17.000)^{1)}$} & \multicolumn{2}{|c|}{$4.677(0.200,8.300)^{1)}$} & \multicolumn{2}{|c|}{$5.321(1.900,8.125)^{1)}$} \\
\hline & & 病程第 12 周 & $60.794(18.300,94.150)^{1)}$ & \multicolumn{2}{|c|}{$11.882(4.500,18.500)^{1)}$} & \multicolumn{2}{|c|}{$5.912(1.300,8.800)^{1)}$} & \multicolumn{2}{|c|}{$6.641(2.800,9.825)^{1)}$} \\
\hline \multirow{3}{*}{ 治疗组 } & \multirow{3}{*}{17} & 治疗前 & $39.024(26.100,54.150)$ & \multicolumn{2}{|l|}{$7.647(8.000,10.500)$} & \multicolumn{2}{|c|}{$3.582(2.000,5.500)$} & \multicolumn{2}{|c|}{$5.012(3.450,6.625)$} \\
\hline & & 病程第 3 周 & $76.177(65.500,91.850)^{17}$ & \multicolumn{2}{|c|}{$14.765(11.000,18.500)^{1)}$} & \multicolumn{2}{|c|}{$7.212(6.500,8.250)^{1 ?}$} & \multicolumn{2}{|c|}{$7.877(6.750,9.675)^{1)}$} \\
\hline & & 病程第 12 周 & $89.959(84.700,99.100)^{17}$ & \multicolumn{2}{|c|}{$18.294(17.000,20.000)^{1)}$} & \multicolumn{2}{|c|}{$8.406(7.600,9.800)^{17}$} & $9.203(8.95$ & $0,10.000)^{12}$ \\
\hline & & & $-0.086^{\mathrm{a}}$ & $-0.208^{\mathrm{a}}$ & & $-0.260^{\mathrm{a}}$ & & -0.8 & $879^{\mathrm{a}}$ \\
\hline$\chi^{2} / t$ 值 & & & $-1.981^{b}$ & $-2.041^{b}$ & & $-2.029^{b}$ & & -2.1 & $194^{b}$ \\
\hline & & & $-2.618^{c}$ & $-2.669^{\circ}$ & & $-2.282^{c}$ & & -2.1 & $139^{\circ}$ \\
\hline & & & $0.931^{\mathrm{a}}$ & $0.835^{\text {a }}$ & & $0.795^{\mathrm{a}}$ & & & $379^{\mathrm{a}}$ \\
\hline$P$ 值 & & & $0.048^{\mathrm{b} 2)}$ & $0.041^{\mathrm{b} 2)}$ & & $0.042^{b 2\rangle}$ & & & $022^{b 2\rangle}$ \\
\hline & & & $0.009^{(2)}$ & $0.008^{\circ 2)}$ & & $0.023^{c 2}$ & & & $032^{\circ 22}$ \\
\hline & & & & & & & & 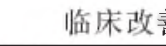 & \\
\hline 组 别 & 例数 & 时间 & 命名 & NIHSS & & $\mathrm{mRS}$ & $\mathrm{AQ}$ 损 & $\begin{array}{l}\text { 提高 } \geqslant 30 \text { 分 } \\
(n, \%)\end{array}$ & $\begin{array}{c}\mathrm{mRS} 0 \sim 2 \\
(n, \%)\end{array}$ \\
\hline & & 治疗前 & $4.100(0.000,7.100)$ & $10.059(5.000,15.500)$ & 3.824 & $(3.000,5.000)$ & & & \\
\hline 对照组 & 17 & 病程第 3 周 & $4.906(0.000,8.600)^{1)} \quad 8$ & $8.235(3.500,13.500)^{1)}$ & 3.235 & $(2.000,4.000)^{1)}$ & & $6(35.29)$ & $11(64.71)$ \\
\hline & & 病程第 12 周 & $5.871(0.050,9.450)^{1)} \quad 5$ & $5.353(1.500,9.000)^{1)}$ & 2.235 & $(1.000,3.500)^{1)}$ & & & \\
\hline & & 治疗前 & $3.212(1.350,4.100)$ & $9.000(6.500,12.000)$ & 4.000 & $(3.500,4.500)$ & & & \\
\hline 治疗组 & 17 & 病程第 3 周 & $7.647(6.550,9.000)^{1)}$ & $4.294(3.000,6.000)^{1)}$ & 2.294 & $(2.000,3.000)^{1)}$ & & $4(82.35)$ & $16(94.12)$ \\
\hline & & 病程第 12 周 & $9.077(8.500,10.000)^{1)} 1$ & $1.765(1.000,2.000)^{1)}$ & & $(1.000,1.000)^{1)}$ & & & \\
\hline & & & $-0.346^{\mathrm{a}}$ & $-0.190^{\mathrm{a}}$ & & $-0.380^{\mathrm{a}}$ & & & \\
\hline$\chi^{2} / t$ 值 & & & $-1.968^{b}$ & $-2.058^{b}$ & & $-2.257^{2)}$ & & & \\
\hline & & & $-2.542^{\circ}$ & $-2.243^{\circ}$ & & $-2.981^{\circ}$ & & & \\
\hline & & & $0.730^{\mathrm{a}}$ & $0.849^{\mathrm{a}}$ & & $0.704^{\mathrm{a}}$ & & & \\
\hline$P$ 值 & & & $0.049^{\mathrm{b} 2)}$ & $0.040^{\mathrm{b} 2)}$ & & $0.024^{\mathrm{b} 2\rangle}$ & & $0.013^{2)}$ & 0.085 \\
\hline & & & $0.011^{\mathrm{c} 2)}$ & $0.025^{\mathrm{c} 2)}$ & & $0.003^{\mathrm{c} 2)}$ & & & \\
\hline
\end{tabular}

注: a. 组间治疗前比较; b. 组间病程第 3 周比较; c. 组间病程第 12 周比较。组内比较, 1) $P<0.05$; 组间比较, 2) $P<0.05$ 。

Note: a. Compared between two groups before treatment; b. Compared between two groups at third week of disease; c. Compared between two groups at 12 th week of disease. Compared within group, 1) $P<0.05$; Compared between two groups, 2 ) $P<0.05$.

法结合常规言语训练更有助于急性脑梗死后皮质 下失语患者言语功能的康复。进而将纳人的 34 例 患者做了言语明显改善的二元回归分析, 结果表明 校正混杂因素影响后, 镜像疗法结合常规言语训练 仍与言语功能明显改善相关, 进一步证实了实施该 治疗方法在改善患者言语功能方面的疗效。其次, 既往有研究发现, 年龄是失语预后良好的危险因素, 年龄增加不利于言语功能的恢复 ${ }^{[30]}$ 。而本研究结果 也证实年龄是言语明显改善的危险因素之一。另 外, 本研究结果表明,2组治疗后不同时间点各指标 评估均较治疗前改善, 说明不管是镜像疗法结合常
规言语训练还是单独常规言语训练均可以改善失 语症患者的言语症状及神经功能。因此, 急性期给 予有效的康复干预能改善脑梗死后患者言语功能 及神经功能缺损, 提示了早期康复的重要性。这一 研究结果与既往的结论一致, 证实了语言障碍康复 的疗效与是否进行语言训练密切相关, 且语言训练 的尽早介人对患者语言功能的恢复有重要意义 ${ }^{[31-32]}$ 。

本研究还发现镜像疗法结合常规言语训练有 助于改善患者神经功能缺损症状, 而目前国内外鲜 见类似报道。考虑由于失语患者接受了 2 周的镜像 治疗直接改善了其言语功能, 而言语功能的改善有 
表 32 组言语功能明显改善的 Logistic 回归分析

Table 3 Logistic regression analysis of speech function improvement

\begin{tabular}{|c|c|c|c|c|c|c|}
\hline \multirow{2}{*}{ 项 目 } & \multicolumn{3}{|c|}{ 单因素 } & \multicolumn{3}{|c|}{ 多因素 } \\
\hline & $O R$ & $95 \% C I$ & $P$ 值 & $O R$ & $95 \% C I$ & $P$ 值 \\
\hline 性别 & 10.556 & $(1.070,104.114)$ & $0.044^{1)}$ & & & \\
\hline 年龄 & 0.904 & $(0.832,0.981)$ & $0.016^{1>}$ & 0.913 & $(0.837,0.997)$ & $0.042^{1>}$ \\
\hline 干预方式 & 8.556 & $(1.736,42.169)$ & $0.008^{17}$ & 7.161 & $(1.262,40.631)$ & $0.026^{17}$ \\
\hline 教育年限 & 1.348 & $(0.953,1.907)$ & 0.091 & & & \\
\hline 高血压 & 0.833 & $(0.203,3.422)$ & 0.800 & & & \\
\hline 糖尿病 & 0.917 & $(0.170,4.930)$ & 0.919 & & & \\
\hline 冠心病 & 0.667 & $(0.082,5.399)$ & 0.704 & & & \\
\hline 脑卒中 & 0.441 & $(0.082,2.387)$ & 0.342 & & & \\
\hline 房颤 & 1.444 & $(0.118,17.671)$ & 0.773 & & & \\
\hline 基底核-内囊区 & 0.571 & $(0.137,2.380)$ & 0.442 & & & \\
\hline 丘脑 & 0.684 & $(0.039,11.949)$ & 0.795 & & & \\
\hline 脑室旁白质 & 0.441 & $(0.082,2.387)$ & 0.342 & & & \\
\hline 基底核十丘脑 & 1.444 & $(0.118,17.671)$ & 0.773 & & & \\
\hline 基底核＋脑室旁白质 & 2.045 & $(0.477,8.774)$ & 0.335 & & & \\
\hline
\end{tabular}

注: 1) $P<0.05$ 。

Note: 1) $P<0.05$.

助于康复训练的正常进行, 进而提高了 NIHSS 和 mRS 的评分。

综上所述, 镜像疗法结合常规言语训练有助于 急性脑梗死后皮质下失语患者言语及神经功能的 康复。同样, 该治疗方法亦与患者 3 个月的言语功 能明显改善相关。并且, 由于急性脑梗死患者病程 早期往往以卧床为主, 而该治疗可以在患者卧位时 进行, 患者依从性好, 还可以和常规言语治疗方法 等相结合进一步提高疗效, 这种简单易行的方法值 得在脑梗死急性失语症患者康复治疗中推广应用。

由于条件限制及样本量较少的原因, 本研究仅 通过临床观察评估疗效, 未涉及该治疗机制方面的 研究。另外, 由于患者对一种新治疗的期望, 急性失 语患者自然恢复程度以及患者出院后继续康复强 度的差异等因素可能会对本研究结果产生影响。因 此, 在今后的研究中应扩大样本量, 同时通过功能 影像学技术探讨其作用机制, 为镜像疗法联合常规 言语训练提高急性脑梗死后皮质下失语的疗效奠 定理论和实践基础。

\section{参考文献}

[1] THIEME H, MORKISCH N, MEHRHOLZ J, et al. Mirror therapy for improving motor function after stroke $[\mathrm{J}]$. Cochrane Database Syst Rev, 2018, 7:CD008449.

[2] STEENWINKEL F, TER MEULEN B, RAMACHANDRAN V. Teaching video neuroimages:mirror therapy against phantom pain [J]. Neurology , 2019,92(9):e1002.

[3] BRODTMANN A, VOGEL A P. Language, meaning, and localization : Last year's words, next year's words [J]. Neurology, 2017, $88(10): 924-925$.

[4] BONIFAZI S, TOMAIUOLO F, ALTOE G, et al. Action observation as a useful approach for enhancing recovery of verb production : new evidence from aphasia [J]. Eur J Phys Rehabil Med, $2013,49: 473-481$.

[5]陈文莉,夏扬, 杨穻, 等. 手动作观察训练对脑卒中失语症患 者语言功能的影响 $[J]$. 中国康复医学杂志, 2014,29(2): 141144.

[6] YOU L, WANG Y, CHEN W, et al. The effectiveness of action observation therapy based on mirror neuron theory in Chinese patients with apraxia of speech after stroke $[\mathrm{J}]$. Eur Neurol, $2019,81(5 / 6): 278-286$.

[7] CHEN W, YE Q,JI X, et al. Mirror neuron system based therapy for aphasia rehabilitation $[\mathrm{J}]$. Front Psychol , 2015, 6:1665.

[8] FROMM D, FORBES M, HOLLAND A, et al. Discourse characteristics in aphasia beyond the Western Aphasia Battery Cutoff $[J]$. Am J Speech Lang Pathol, 2017, 26(3): 762-768.

[9] WU J B, LYU Z H, LIU X J, et al. Development and standardization of a new cognitive assessment test battery for Chinese aphasic patients:a preliminary study $[\mathrm{J}]$. Chin Med J (Engl), $2017,130(19): 2283-2290$.

[10 ] CHEN W L,YE Q,ZHANG S C,et al. Aphasia rehabilitation based on mirror neuron theory: a randomized-block-design study of neuropsychology and functional magnetic resonance imaging $[\mathrm{J}]$. Neural Regen Res, 2019,14(6):1004-1012.

[11] STEFANIAK J D, HALAI A D, LAMBON RALPH M A. The neural and neurocomputational bases of recovery from post- 
stroke aphasia $[J]$. Nat Rev Neurol, 2020, 16(1):43-55.

[12] REN C, ZHANG G, XU X, et al. The effect of rTMS over the different targets on language recovery in stroke patients with global aphasia : a randomized sham-controlled study $[\mathbf{J}]$. Biomed Res Int , 2019,2019:4589056.

[13] 陈莺, 李焰生, 王智樱, 等. 罗奈哌齐治疗卒中后失语的疗效 观察 $[J]$. 中华内科杂志, 2010,49(2):115-118.

[14] KANG E K, SOHN H M, HAN M K, et al. Subcortical aphasia after stroke [J]. Ann Rehabil Med, 2017,41(5):725-733.

[15] 单春雷, 于美霞. 皮质下失语症的特点及其相关机制探讨 [ J J . 中国临床康复, 2004,8(4):720-721.

[16] 李素梅,崔成立, 高风霞. 皮质下失语症产生机制的研究现 状 $[J]$. 中国康复医学杂志, 2010,25(4):382-385.

[17] KULJIC-OBRADOVIC D. Subcortical aphasia:three different language disorder syndromes? [J]. Eur J Neurol, 2003, 10(4): $445-448$.

[18] MAESHIMA S,OKAMOTO S,OKAZAKI H, et al. Aphasia following left putaminal hemorrhage at a rehabilitation hospital $[\mathrm{J}]$. Eur Neurol ,2018,79(1/2):33-37.

[19] GANESAN R V, VEERASAMY K K, CHITTALA M, et al. Thalamic abscess caused by a rare pathogen-streptococcus sanguinisA report and a review on thalamic abscess $[\mathrm{J}]$. Neurol India, $2019,67(3): 875-879$.

[20] SCHMAND B,DE BIE R,KONING-HAANSTRA M, et al. Unilateral pallidotomy in PD:A controlled study of cognitive and behavioral effects $[\mathrm{J}]$. Neurology, 2000,54(5):1058-1064.

[21] RADANOVIC M, MANSUR L L. Aphasia in vascular lesions of the basal ganglia: A comprehensive review $[\mathrm{J}]$. Brain Lang, 2017, $173: 20-32$.

[22] CAPLAN D, WATERS G, KENNEDY D, et al. A study of syn-

tactic processing in aphasia II :neurological aspects $[\mathrm{J}]$. Brain Lang, 2007, 101(2):151-177.

[23] HENSELER I, REGENBRECHT F, OBRIG H. Lesion correlates of patholinguistic profiles in chronic aphasia:comparisons of syndrome-, modality- and symptom-level assessment $[\mathbf{J}]$. Brain, 2014, 137 (Pt 3):918-930.

[24] BURGALETA M, SANJUAN A, VENTURA-CAMPOS N, et al. Bilingualism at the core of the brain. Structural differences between bilinguals and monolinguals revealed by subcortical shape analysis $[\mathrm{J}]$. Neuroimage, $2016,125: 437-445$.

[25] CHEN Y, LANDIN-ROMERO R, KUMFOR F, et al. Cerebellar structural connectivity and contributions to cognition in frontotemporal dementias [J]. Cortex, 2020,129:57-67.

[26] 姚婧璠,陈红燕, 张玉梅. 弥散张量成像对皮质下失语症发病 机制的研究 $[J]$. 中国康复理论与实践, 2018,24(8):869-879.

[27] 汪凯, 卡兴华, 王长青, 等. 皮层下脑血管病后言语障碍的康 复和预后 $[\mathrm{J}]$. 中国康复医学杂志, 2001, 16(3):149-151.

[28] FINSTERWALDER S,DEMEYERE N, GLLEBERT C R. Deficit in feature-based attention following a left thalamic lesion $[\mathrm{J}]$. Neuropsychologia, 2017, 102:1-10.

[29］程熙,赖靖慧,王志峰, 等. 多奈哌齐治疗脑卒中后基底节 性失语症的疗效观察 $[\mathrm{J}]$. 康复学报, 2015,25(4): 34-36.

[30] 王美霞, 李明. 皮质下失语症 $[\mathrm{J}]$. 临床神经病学杂志, 2009, 22(3):229-230.

[31] 孔亚泞, 丘卫红, 胡昔权, 等. 脑卒中后基底核性失语特征及 针对性语言训练的效应 $[\mathrm{J}]$. 中国临床康复, 2004,8 (25): $5216-5217$.

[32] 丘卫红, 突祖林, 万桂芳, 等. 无障碍电脑语言系统 U1 在失 语症患者语言训练中的应用 $[\mathrm{J}]$. 中国临床康复, 2004, 8 (1): $28-29$.

\title{
Therapeutic Effect of Mirror Therapy Combined with Conventional Speech Training on Subcortical Aphasia after Acute Cerebral Infarction
}

\author{
CHEN Qingmei, CAI Xiuying, KE Jun, SHEN Dan, FAN Keke, YANG Weixin, LI Li, FANG Qi* \\ The First Affliated Hospital of Soochow University, Suzhou, Jiangsu 215000, China \\ * Correspondence: FANG Qi, E-mail: fangqi_008@126.com
}

\begin{abstract}
Objective: To observe the effect of combined mirror therapy and conventional speech training on the rehabilitation of subcortical aphasia after acute cerebral infarction. Methods: A total of thirty-four patients with subcortical aphasia after cerebral infarction were randomly divided into treatment group and control group. The treatment group received conventional speech therapy combined with mirror therapy, and patients in the control group were only given conventional speech therapy. Both groups received conventional speech therapy for acute cerebral infarction on the 7th day after onset. The aphasia quotient (AQ) in the western aphasia test, National Institutes of Health Stroke Scale (NIHSS) and the Modified Rankin Scale (mRS) were assessed before treatment, and at the third and 12 th week of disease. AQ which increased $\geqslant 30$ scores within three months was regarded as significant improvement of speech function, and mRS scores of 0-2 were considered to have a good prognosis for neurological function. Results: There was no significant difference between the two groups before the treatment in all the indicators $(P>0.05)$. After 2 weeks' treatment, NIHSS, AQ, and $\mathrm{AQ}$ (spontaneous speech, listening comprehension, retelling and naming) and $\mathrm{mRS}$ scores of the two groups were improved at the third and 12th week of disease, which were statistically significalt differences compared with before treatment $(P<0.05)$. The scores of aphasia, spontaneous speech, listening comprehension, retelling, naming. NIHSS and mRS in the treatment group at the third and $12 \mathrm{th}$ week of disease were better than those in the control group $(P<0.05)$. The significant improvement rate of speech function was better in the treatment group $(82.35 \%)$ than that in the control group $(35.29 \%)(P=0.013)$. After adjusting for confounding factors, the intervention factor and age were correlated with aphasia function significant improvement $(P<0.05)$. Conclusion: Mirror therapy combined with conventional speech therapy can help speech and neurological function rehabilitation after acute cerebral infarction in patients with subcortical aphasia, and can also promote the significant improvement of speech function in 3 months.
\end{abstract}

KEY WORDS acute cerebral infarction; subcortical aphasia; mirror therapy; conventional speech training; speech function DOI: $10.3724 /$ SP.J.1329.2020.03010 